\title{
Auswahlbibliografie Neuerscheinungen
}

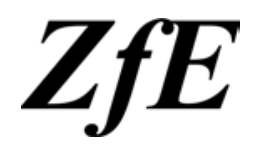

\section{Besprechung vorbehalten}

- Timm Albers: Sprache und Integration im Kindergarten. Eine quantitativ-qualitative Analyse der sprachlichen und kommunikativen Kompetenzen von drei- bis sechsjährigen Kindern. Bad Heilbrunn/Obb.: Klinkhardt 2009. 282 S. ISBN 978-3-78151682-3. Preis: $32,-€$.

- Peter Alheit/Heide von Felden (Hrsg.): Lebenslanges Lernen und erziehungswissenschaftliche Biographieforschung. Konzepte und Forschung im europäischen Diskurs. Wiesbaden: VS Verlag für Sozialwissenschaften 2009. 245 S. ISBN 978-3531-15600-2. Preis: 29,90€.

- Karin Altgeld/Sybille Stöbe-Blossey (Hrsg.): Qualitätsmanagement in der frühkindlichen Bildung, Erziehung und Betreuung. Perspektiven für eine öffentliche Qualitätspolitik. Wiesbaden: VS Verlag für Sozialwissenschaften 2009. 264 S. ISBN 978-3-531-16008-5. Preis: 29,90€.

- Marco Althaus: Die ANTI-Harvards. Wie Bildungskonzerne Amerikas Hochschulwesen revolutionieren. Berlin: LIT 2009. 848 S. ISBN 978-3-8258-1946-0. Preis: $29,90 €$.

- Hans-Jürgen Appelrath/Leonore Schulze (Hrsg.): Auf dem Weg zu exzellentem ELearning. Vernetzung und Kooperation der Hochschullehre in Niedersachsen. Münster: Waxmann 2009. 256 S. ISBN 978-3-8309-2122-6. Preis: 24,90€.

- Karl-Heinz Arnold/Sigrid Blömeke/Rudolf Messner/Jörg Schlömerkemper: Allgemeine Didaktik und Lehr-Lernforschung. Kontroversen und Entwicklungsperspektiven einer Wissenschaft vom Unterricht. Bad Heilbrunn/Obb.: Klinkhardt 2009. 212 S. ISBN 978-3-7815-1674-8. Preis: 19,90€.

- Sylke Bartmann/Axel Fehlhaber/Sandra Kirsch/Wiebke Lohfeld (Hrsg.): „Natürlich stört das Leben ständig“. Perspektiven auf Entwicklung und Erziehung. Wiesbaden: VS Verlag für Sozialwissenschaften 2009. 185 S. ISBN 978-3-531-16609-4. Preis: $29,90 €$.

- Roland Becker-Lenz/Stefan Busse/Gudrun Ehlert/Silke Müller (Hrsg.): Professionalität in der Sozialen Arbeit. Standpunkte, Kontoversen, Perspektiven. Wiesbaden: VS Verlag für Sozialwissenschaften 2009. 352 S. ISBN 978-3-531-16079-5. Preis: $39,90 €$.

- Christ of Beckmann: Qualitätsmanagement und Soziale Arbeit. Wiesbaden: VS Verlag für Sozialwissenschaften 2009. 221 S. ISBN 978-3-531-16799-2. Preis: $24,90 €$. 
- Nils Berkemeyer/Harm Kuper/Veronika Manitius/Kathrin Müthing (Hrsg.): Schulische Vernetzung. Eine Übersicht zu aktuellen Netzwerkprojekten. Münster: Waxmann 2009. 188 S. ISBN 978-3-8309-2113-4. Preis: 24,90€.

- Ralf Biermann: Der mediale Habitus von Lehramtsstudierenden. Eine quantitative Studie zum Medienhandeln angehender Lehrpersonen. Wiesbaden: VS Verlag für Sozialwissenschaften 2009. 279 S. ISBN 978-3-531-16520-2. Preis: 34,90€.

- Christine Böckelmann: Arbeitsplatz Hochschule. Vom Allrounder-Anspruch zum kompetenzbasierten Personalmanagement. Münster: Waxmann 2009. 180 S. ISBN 978-3-8309-2108-0. Preis: 24,90€.

- Thorsten Bohl/Hanna Kiper (Hrsg.): Lernen aus Evaluationsergebnissen. Verbesserungen planen und implementieren. Bad Heilbrunn/Obb.: Klinkhardt 2009. $309 \mathrm{~S}$. ISBN 978-3-7815-1677-9. Preis: 19,90€.

- Wilfried Bos/Martin Bonsen/Nicole Kummer/Katrin Lintorf/Kristina Frey (Hrsg.): TIMSS 2007. Dokumentation der Erhebungsinstrumente zur Trends in International Mathematics and Science Study. Münster: Waxmann 2009. 240 S. ISBN 978-3-83092143-1. Preis: 59,- $€$.

- Peter J. Brenner: Wie Schule funktioniert. Schüler, Lehrer, Eltern im Lernprozess. Stuttgart: Kohlhammer 2009. 267 S. ISBN 978-3-17-019519-6. Preis: 24,80€.

- Michael Brodowski/Ulrike Devers-Kanoglu/Bernd Overwien/Matthias Rohs/Susanne Salinger/Manfred Walser (Hrsg.): Informelles Lernen und Bildung für nachhaltige Entwicklung. Beiträge aus Theorie und Praxis. Opladen: Barbara Budrich 2009. 275 S. ISBN 978-3-86649-233-2. Preis: 29,90€.

- Florence Buchmann: Schulentwicklung verstehen. Die soziale Konstruktion des Wandels (Internationale Hochschulschriften, Bd. 526). Münster: Waxmann 2009. 310 S. ISBN 978-3-8309-2126-4. Preis: 34,90€.

- Carsten Bünger/Peter Euler/Andreas Gruschka/Ludwig A. Pongratz (Hrsg.): Heydorn lesen! Herausforderungen kritischer Bildungstheorie. Paderborn: Schöningh 2009. 238 S. ISBN 978-3-506-76768-4. Preis: 29,90€.

- Alois Bürli/Urs Strasser/Anne-Dore Stein (Hrsg.): Integration aus internationaler Sicht. Bad Heilbrunn/Obb.: Klinkhardt 2009. 336 S. ISBN 978-3-7815-1558-1. Preis: $22,90 €$.

- Carl-Peter Buschkühle/Ludwig Duncker/Vadim Oswalt (Hrsg.): Bildung zwischen Standardisierung und Heterogenität - ein interdisziplinärer Diskurs. Wiesbaden: VS Verlag für Sozialwissenschaften 2009. 237 S. ISBN 978-3-531-16800-5. Preis: $29,90 €$.

- Heike de Boer/Heike Deckert-Peaceman (Hrsg.): Kinder in der Schule. Zwischen Gleichaltrigenkultur und schulischer Ordnung. Wiesbaden: VS Verlag für Sozialwissenschaften 2009. 330 S. ISBN 978-3-531-15935-5. Preis: 34,90€.

- Claudia Dehn (Hrsg.): Pädagogische Qualität. Einflussfaktoren und Wirkungsmechanismen (Schriftenreihe für kritische Sozialforschung und Bildungsarbeit, Bd. 15). Hannover: Expressum-Verlag 2009. 156 S. ISBN 978-3-89069-020-9. Preis: $20,-€$.

- İnci Dirim/Paul Mecheril (Hrsg.): Migration und Bildung. Soziologische und erziehungswissenschaftliche Schlaglichter. Münster: Waxmann 2009. 292 S. ISBN 978-3-8309-2109-7. Preis: 29,90€. 
- Peter Dudek: „Versuchsacker für eine neue Jugend“. Die Freie Schulgemeinde Wickersdorf 1906-1945. Bad Heilbrunn/Obb.: Klinkhardt 2009. 480 S. ISBN 978-37815-1681-6. Preis: 29,90€.

- Ulrike Eichinger: Zwischen Anpassung und Ausstieg. Perspektiven von Beschäftigten im Kontext der Neuordnung Sozialer Arbeit. Wiesbaden: VS Verlag für Sozialwissenschaften 2009. 238 S. ISBN 978-3-531-16473-1. Preis: 34,90€.

- Helmut Fend/Fred Berger/Urs Grob (Hrsg.): Lebensverläufe, Lebensbewältigung, Lebensglück. Ergebnisse der LifE-Studie. Wiesbaden: VS Verlag für Sozialwissenschaften 2009. 482 S. ISBN 978-3-531-15352-0. Preis: 39,90€.

- Jonas Flöter/Christian Ritzi (Hrsg.): Das Joachimsthalsche Gymnasium. Beiträge zum Aufstieg und Niedergang der Fürstenschule der Hohenzollern. Bad Heilbrunn/Obb.: Klinkhardt 2009. 315 S. ISBN 978-3-7815-1655-7. Preis: 24,90€.

- Ulrich Gebhard: Kind und Natur. Die Bedeutung der Natur für die psychische Entwicklung. 3. überarb. u. erw. Aufl. Wiesbaden: VS Verlag für Sozialwissenschaften 2009. 313 S. ISBN 978-3-531-16338-3. Preis: 39,90€.

- Brigitte Geldermann/Sabine Seidel/Eckart Severing: Rahmenbedingungen zur Anerkennung informell erworbener Kompetenzen. Bielefeld: W. Bertelsmann 2009. 265 S. ISBN 978-3-7639-3884-1. Preis: 24,90€.

- Jürgen Gößling: Selbstverhältnisse als Gegenstand der Erziehungswissenschaft. Zur Logik pädagogischen Handelns. Berlin: LIT 2008. ISBN 978-3-8258-1676-6. Preis: $19,90 €$.

- Ingrid Gogolin/Ursula Neumann (Hrsg.): Streitfall Zweisprachigkeit - The Bilingualism Controversy. Wiesbaden: VS Verlag für Sozialwissenschaften 2009. 338 S. ISBN 978-3-531-15886-0. Preis: 29,90€.

- Heinrich Greving/Petr Ondracek (Hrsg.): Spezielle Heilpädagogik. Eine Einführung in die handlungsorientierte Heilpädagogik. Stuttgart: Kohlhammer 2009. 268 S. ISBN 978-3-17-020014-2. Preis: 24,- €.

- Frauke Grittner: Leistungsbewertung mit Portfolio in der Grundschule. Eine mehrperspektivische Fallstudie aus einer notenfreien sechsjährigen Grundschule. Bad Heilbrunn/Obb.: Klinkhardt 2009. 205 S. ISBN 978-3-7815-1629-8. Preis: 29,80€.

- Hatice Gündoğdu/Ulrike Zenk: Kampf der Kulturen? Zwei Frauen gestalten Integration. Norderstedt: Books on Demand 2008. 148 S. ISBN 978-3-8370-4961-9. Preis: $12,90 €$.

- Rahim Hajji: Sozialisationsprozesse in Familien mit marokkanischem Migrationshintergund. Opladen: Budrich UniPress 2009. 234 S. ISBN 978-3-940755-26-1. Preis: 24,90€.

- Franz Hamburger: Abschied von der Interkulturellen Pädagogik. Plädoyer für einen Wandel sozialpädagogischer Konzepte. Weinheim: Juventa 2009. 212 S. ISBN 9783-7799-1229-3. Preis: 19,50€.

- Florian Heinrich/Ernst Plaum: Fragwürdige Fragebögen. Paradigmatische Untersuchungen zur Gewalt in der Schule. Wiesbaden: VS Verlag für Sozialwissenschaften 2009. 180 S. ISBN 978-3-531-16534-9. Preis: 29,90€.

- Werner Helsper/Rolf-Torsten Kramer/Merle Hummrich/Susann Busse: Jugend zwischen Familie und Schule. Eine Studie zu pädagogischen Generationsbeziehungen. 
Wiesbaden: VS Verlag für Sozialwissenschaften 2009. 440 S. ISBN 978-3-53116574-5. Preis: 39,90€.

- Remi Hess: Die Praxis des Tagebuchs. Beobachtung - Dokumentation - Reflexion. Münster: Waxmann 2009. 152 S. ISBN 978-3-8309-1998-8. Preis: 16,90€.

- Ilona Heuchel/Eva Lindner/Karin Sprenger (Hrsg.): Familienzentren in NordrheinWestfalen. Beispiele innovativer Praxis. Münster: Waxmann 2009. 226 S. ISBN 9783-8309-2106-6. Preis: 19,90€.

- Andreas Hoffmann-Ocon: Schule zwischen Stadt und Staat. Steuerungskonflikte zwischen städtischen Schulträgern, höheren Schulen und staatlichen Unterrichtsbehörden im 19. Jahrhundert. Bad Heilbrunn/Obb.: Klinkhardt 2009. 278 S. ISBN 978-3-78151656-4. Preis: $34,-€$.

- Andreas Hoffmann-Ocon/Dominique Oesch: „Die Deutsche Schule“ im Nationalsozialismus. 10. Beiheft zu die Deutsche Schule. Münster: Waxmann 2009. 144 S. ISBN 978-3-8309-2151-6. Preis: 14,-€.

- Johanna Hopfner: Gelegentliche Gedanken über Erziehung (Erziehung in Wissenschaft und Praxis, Bd. 1). Frankfurt am Main: Peter Lang 2008. 198 S. ISBN 978-3631-55167-7. Preis: 29,80€.

- Johanna Hopfner/Andreás Németh (Hrsg.): Pädagogische und kulturelle Strömungen in der k. u. k. Monarchie. Lebensform, Herbartianismus und reformpädagogische Bewegungen (Erziehung in Wissenschaft und Praxis, Bd. 2). Frankfurt am Main: Peter Lang 2008. 200 S. ISBN 978-3-631-56719-7. Preis: 39,-€.

- Sabine Hornberg/Inci Dirim/Gregor Lang-Wojtasik/Paul Mecheril (Hrsg.): Beschreiben - Verstehen - Interpretieren. Stand und Perspektiven International und Interkulturell Vergleichender Erziehungswissenschaft in Deutschaland. Münster: Waxmann 2009. 202 S. ISBN 978-3-8309-2128-8. Preis: 24,90€.

- Christine Hunner-Kreisel/Arne Schäfer/Matthias D. Witte (Hrsg.): Jugend, Bildung und Globalisierung. Sozialwissenschaftliche Reflexion in internationaler Perspektive. Weinheim: Juventa 2008. 248 S. ISBN 978-3-7799-1749-6. Preis: 22,-€.

- Sylvia Kade: Altern und Bildung. Eine Einführung. 2., akt. und überarb. Aufl. Bielefeld: W. Bertelsmann 2009. 253 S. ISBN 978-3-7639-3336-5. Preis: 24,90€.

- Paul Kellermann/Manfred Boni/Elisabeth Meyer-Renschhausen (Hrsg.): Zur Kritik europäischer Hochschulpolitik. Forschung und Lehre unter Kuratel betriebswirtschaftlicher Denkmuster. Wiesbaden: VS Verlag für Sozialwissenschaften 2009. 240 S. ISBN 978-3-531-16314-7. Preis: 39,90€.

- Mouhanad Khorchilde: Der islamische Religionsunterricht zwischen Integration und Parallelgesellschaft. Einstellungen der islamischen ReligionslehrerInnen an öffentlichen Schulen. Wiesbaden: VS Verlag für Sozialwissenschaften 2009. 195 S. ISBN 978-3-531-16493-9. Preis: 29,90€.

- Svetlana Kiel: Wie deutsch sind Russlanddeutsche? Eine empirische Studie zur ethnisch-kulturellen Identität in russlanddeutschen Aussiedlerfamilien. Münster: Waxmann 2009. 216 S. ISBN 978-3-8309-2068-7. Preis: 24,90€.

- Leopold Klepacki/Andreas Schröer/Jörg Zirfas (Hrsg.): Der Alltag der Kultivierung. Studien zu Schule, Kunst und Bildung. Münster: Waxmann 2009. 216 S. ISBN 9783-8309-2087-8. Preis: 24,90€. 
- Michaela Knust/Anke Hanft (Hrsg.): Weiterbildung im Elfenbeinturm!? Münster: Waxmann 2009. 224 S. ISBN 978-3-8309-2059-5. Preis: 29,90€.

- Mareike Kobarg: Unterstützung unterrichtlicher Lernprozesse aus zwei Perspektiven. Eine Gegenüberstellung. Münster: Waxmann 2009. 234 S. ISBN 978-3-8309-2114-1. Preis: $24,90 €$.

- Sascha Koch/Michael Schemmann (Hrsg.): Neo-Institutionalismus in der Erziehungswissenschaft. Grundlegende Texte und empirische Studien. Wiesbaden: VS Verlag für Sozialwissenschaften 2009. 360 S. ISBN 978-3-531-16290-4. Preis: 39,90€.

- Johannes Kopp: Bildungssoziologie. Eine Einführung anhand empirischer Studien. Wiesbaden: VS Verlag für Sozialwissenschaften 2009. 212 S. ISBN 978-3-53114093-3. Preis: 14,90€.

- Susanne Kraft/Wolfgang Seitter/Lea Kollewe: Professionalitätsentwicklung des Weiterbildungspersonals. Bielefeld: W. Bertelsmann 2009. 108 S. ISBN 978-3-76391972-7. Preis: $16,90 €$.

- Roland Lauterbach/Hartmut Giest/Brunhilde Marquardt-Mau (Hrsg.): Lernen und kindliche Entwicklung. Elementarbildung und Sachunterricht. Bad Heilbrunn/Obb.: Klinkhardt 2009. 286 S. ISBN 978-3-7815-1675-5. Preis: 16,- $€$.

- Drorit Lengyel: Zweitspracherwerb in der Kita. Eine integrative Sicht auf die sprachliche und kognitive Entwicklung mehrsprachiger Kinder (Internationale Hochschulschriften, Bd. 521). Münster: Waxmann 2009. 284 S. ISBN 978-3-8309-2086-1. Preis: $29,90 €$.

- Miriam Leuchter: Die Rolle der Lehrperson bei der Aufgabenbearbeitung. Unterrichtsbezogene Kognitionen von Lehrpersonen (Empirische Erziehungswissenschaft, Bd. 13). Münster: Waxmann 2009. 312 S. ISBN 978-3-8309-2115-8. Preis: 29,90€.

- Arnold Lohaus/Holger Domsch (Hrsg.): Psychologische Förder- und Interventionsprogramme für das Kindes- und Jugendalter. Heidelberg: Springer 2009. 336 S. ISBN 978-3-540-88383-8. Preis: 39,95€.

- Cornelia Maier-Gutheil: Zwischen Beratung und Begutachtung. Pädagogische Professionalität in der Existenzgründungsberatung. Wiesbaden: VS Verlag für Sozialwissenschaften 2009. 272 S. ISBN 978-3-531-16588-2. Preis: 34,90€.

- Ulrike Manz/Bruno Schmid (Hrsg.): Bioethik in der Schule. Grundlagen und Gestaltungsformen. Münster: Waxmann 2009. 184 S. ISBN 978-3-8309-2127-1. Preis: $24,90 €$.

- Gudrun Marci-Boehncke/Matthias Rath (Hrsg.): Jugend - Werte - Medien: Das Modell. Weinheim: Beltz 2009. 207 S. ISBN 978-3-407-25512-9. Preis: 29,95€.

- Aila-Leena Matthies/Ehrenhard Skiera (Hrsg.): Das Bildungswesen in Finnland. Bad Heilbrunn/Obb.: Klinkhardt 2009. 292 S. ISBN 978-7815-1678-6. Preis: 19,90€.

- Gerhard Mertens/Ursula Frost/Volker Ladenthin/Winfried Böhm (Hrsg.): Handbuch der Erziehungswissenschaft, Bd. 2: Schule, Erwachsenenbildung, Weiterbildung. Paderborn: Schöningh 2009. 1284 S. ISBN 978-3-506-76496-6. Preis: 118,-€.

- Astrid Messerschmidt: Weltbilder und Selbstbilder. Bildungsprozesse im Umgang mit Globalisierung, Migration und Zeitgeschichte. Frankfurt am Main: Brandes \& Apsel 2009. 280 S. ISBN 978-3-86099-395-8. Preis: 29,90€.

- Christine Meyer/Michael Tetzer/Katharina Rensch (Hrsg.): Liebe und Freundschaft in der Sozialpädagogik. Personale Dimension professionellen Handelns. Wiesbaden: 
VS Verlag für Sozialwissenschaften 2009. 298 S. ISBN 978-3-531-16406-9. Preis: $34,90 €$.

- Sascha Neumann/Philipp Sandermann (Hrsg.): Kultur und Bildung. Neue Fluchtpunkte für die sozialpädagogische Forschung? Wiesbaden: VS Verlag für Sozialwissenschaften 2009. 238 S. ISBN 978-3-531-16193-8. Preis: 29,90€.

- Norbert Neuß: Biographisch bedeutsames Lernen. Empirische Studien über Lerngeschichten in der Lehrerbildung (Studien zur Bildungsgangforschung, Bd. 25). Opladen: Barbara Budrich 2009. 428 S. ISBN 978-3-86649-242-4. Preis: 42,-€.

- Melanie Oechler: Dienstleistungsqualität in der Sozialen Arbeit. Eine rhetorische Modernisierung. Wiesbaden: VS Verlag für Sozialwissenschaften 2009. 204 S. ISBN 978-3-531-16528-8. Preis: 29,90€.

- Bernd Overwien/Hanns-Fred Rathenow (Hrsg.): Globalisierung fordert politische Bildung. Politisches Lernen im globalen Kontext. Opladen: Barbara Budrich 2009. 302 S. ISBN 978-3-86649-222-6. Preis: 26,90€.

- Mona Pielorz: Personalentwicklung und Mitarbeiterführung in Weiterbildungseinrichtungen. Bielefeld: W. Bertelsmann 2009. 110 S. ISBN 978-3-7639-1965-9. Preis: $18,90 €$.

- Ludwig A. Pongratz: Bildung im Bermuda-Dreieck: Bologna - Lissabon - Berlin. Eine Kritik der Bildungsreform. Paderborn: Schöningh 2009. 170 S. ISBN 978-3506-76728-8. Preis: 22,90€.

- Ludwig A. Pongratz: Untiefen im Mainstream. Zur Kritik konstruktivistisch-systemtheoretischer Pädagogik. Paderborn: Schöningh 2009. 228 S. ISBN 978-3-506-767424. Preis: $24,90 €$.

- Aglaia Przyborski/Monika Wohlrab-Sahr: Qualitative Sozialforschung. Ein Arbeitsbuch. 2. korr. Aufl. München: Oldenbourg 2009. 403 S. ISBN 978-3-486-59103-3. Preis: $34,80 €$.

- Günter Ratschinski: Selbstkonzept und Berufswahl. Eine Überprüfung der Berufswahltheorie von Gottfredson an Sekundarschülern. Münster: Waxmann 2009. 236 S. ISBN 978-3-8309-2101-1. Preis: 25,50€.

- Hans H. Reich: Zweisprachige Kinder. Sprachaneignung und sprachliche Fortschritte im Kindergartenalter (Interkulturelle Bildungsforschung, Bd. 16). Münster: Waxmann 2009. 272 S. ISBN 978-3-8309-2137-0. Preis: 34,90€.

- Jeanette Roos/Hermann Schöler(Hrsg.): Entwicklung des Schriftspracherwerbs in der Grundschule. Längsschnittanalyse zweier Kohorten über die Grundschulzeit. Wiesbaden: VS Verlag für Sozialwissenschaften 2009. 252 S. ISBN 978-3-531-16538-7. Preis: 29,90€.

- Anne Rosken: Diversity und Profession. Eine biographisch-narrative Untersuchung im Kontext der Bildungssoziologie. Wiesbaden: VS Verlag für Sozialwissenschaften 2009. 292 S. ISBN 978-3-531-16653-7. Preis: 34,90€.

- Michael Schabdach: Soziale Konstruktionen des Drogenkonsums und Soziale Arbeit. Historische Dimensionen und aktuelle Entwicklungen. Wiesbaden: VS Verlag für Sozialwissenschaften 2009. 283 S. ISBN 978-3-531-16752-7. Preis: 39,90€.

- Albert Scherr: Jugendsoziologie. Einführung in Grundlagen und Theorien. 9. erw. und umfassend überarb. Aufl. Wiesbaden: VS Verlag für Sozialwissenschaften 2009. 246 S. ISBN 978-3-531-16480-9. Preis: 19,90€. 
- Christine Schmidt: Nachhaltigkeit lernen? Der Diskurs um Bildung für nachhaltige Entwicklung aus der Sicht evaluationstheoretischer Anthropologie. Opladen: Barbara Budrich 2009. 221 S. ISBN 978-3-86649-241-7. Preis: 24,90€.

- Wilfried Schubarth/Karsten Speck (Hrsg.): Regionale Abwanderung Jugendlicher. Theoretische Analysen, empirische Befunde und politische Gegenstrategien. Weinheim: Juventa 2009. 260 S. ISBN 978-3-7799-1750-2. Preis: 26,-€.

- Isabel Sievers: Individuelle Wahrnehmung, nationale Denkmuster. Einstellungen deutscher und französischer Lehrkräfte zu Heterogenität im Unterricht. Frankfurt am Main: Brandes \& Apsel 2009. 208 S. ISBN978-3-85099-356-9. Preis: 19,90€.

- Margrit Stamm: Begabte Minoritäten. Wiesbaden: VS Verlag für Sozialwissenschaften 2009. 250 S. ISBN 978-3-531-16104-4. Preis: 29,90€.

- Gabriele Strobel-Eisele/Albrecht Wacker (Hrsg.): Konzepte des Lernens in der Erziehungswissenschaft. Phänomene, Reflexionen, Konstruktionen. Bad Heilbrunn/ Obb.: Klinkhardt 2009. 172 S. ISBN 978-3-7815-1669-4. Preis: 32,- $€$.

- Ina ter Avest (Hrsg.): Education in Conflict (Religious Diversity and Education in Europe, Bd. 9). Münster: Waxmann 2009. 140 S. ISBN 978-3-8309-2072-4. Preis: $19,90 €$.

- Marc Thielen: Wo anders leben. Migration, Männlichkeit und Sexualität. Biografische Interviews mit iranischstämmigen Migranten in Deutschland. Münster: Waxmann 2009. 281 S. ISBN 978-3-8309-2094-6. Preis: 29,90€.

- Rudolf Tippelt/Aiga von Hippel (Hrsg.): Handbuch Erwachsenenbildung/Weiterbildung. 3. überarb. u. erw. Aufl. Wiesbaden: VS Verlag für Sozialwissenschaften 2009. 1105 S. ISBN 978-3-531-15506-7. Preis: 79,90€.

- Rudolf Tippelt/Bernhard Schmidt/Simone Schnurr/Simone Sinner/Catharina Theisen: Bildung Älterer. Chancen im demografischen Wandel. Bielefeld: W. Bertelsmann 2009. 223 S. ISBN 978-3-7639-1971-0. Preis: 44,90€.

- Agnes Trattner: Piercing, Tattoo und Schönheitsoperationen. Jugendliche Protesthaltung oder psychopathologische Auffälligkeit? Frankfurt am Main: Peter Lang 2008. 156 S. ISBN 978-3-631-57666-3. Preis: 34,- $€$.

- Pille Valk/Gerdien Bertram-Troost/Markus Friederici/Céline Béraud (Eds.): Teenagers' Perspectives on the Role of Religion in their Lives, Schools and Societies. A European Quantitative Study. Münster: Waxmann 2009. 448 S. ISBN 978-3-83092118-9. Preis: $34,90 €$.

- vbw - Vereinigung der Bayerischen Wirtschaft e.V. (Hrsg.): Geschlechtsdifferenzen im Bildungssystem. Jahresgutachten 2009 des Aktionsrats Bildung. Wiesbaden: VS Verlag für Sozialwissenschaften 2009. 196 S. ISBN 978-3-531-16463-2. Preis: $24,90 €$.

- Anna van der Want/Cok Bakker/Ina ter Avest/Judith Everington (Eds.): Teachers Responding to Religious Diversity in Europe. Researching Biography and Pedagogy. Münster: Waxmann 2009. 211 S. ISBN 978-3-8309-2119-6. Preis: 24,90 €.

- Michael Wimmer/Roland Reichenbach/Ludwig A. Pongratz (Hrsg.): Medien, Technik und Bildung. Paderborn: Schöningh 2009. 177 S. ISBN 978-3-506-76741-7. Preis: $24,90 €$. 
- Susanne Weinert Portmann: Familie - ein Symbol der Kultur. Perspektiven sozialpädagogischer Arbeit mit Familien. Wiesbaden: VS Verlag für Sozialwissenschaften 2009. 193 S. ISBN 978-3-531-16610-0. Preis: 24,90€.

- Jürgen Wittpoth: Einführung in die Erwachsenenbildung. 3. überarb. Aufl. Opladen: Barbara Budrich 2009. 224 S. ISBN 978-3-8252-8244-8. Preis: 14,90€.

- Christine Ziegler: Partizipation der Schüler im naturwissenschaftlichen Fachunterricht (Studien zur Bildungsgangforschung, Bd. 19). Opladen: Barbara Budrich 2009. 348 S. ISBN 978-3-86649-127-4. Preis: 36,-€.

- Anja Zwingenberger: Wirksamkeit multimedialer Lernmaterialien. Kritische Bestandsaufnahme und Metaanalyse empirischer Evaluationsstudien (Pädagogische Psychologie und Entwicklungspsychologie, Bd. 73). Münster: Waxmann 2009. 218 S. ISBN 978-3-8309-2147-9. Preis: 25,50€. 